\title{
Supersymmetric traversable wormholes
}

\author{
Andrés Anabalón, ${ }^{a}$ Bernard de Wit ${ }^{b, c}$ and Julio Oliva ${ }^{d}$ \\ ${ }^{a}$ Departamento de Ciencias, Facultad de Artes Liberales, Universidad Adolfo Ibáñez, \\ Avenida Padre Hurtado 750, Viña del Mar, Chile \\ ${ }^{b}$ Institute for Theoretical Physics, Utrecht University, \\ Princetonplein 5, 3584 CC Utrecht, The Netherlands \\ ${ }^{c}$ Nikhef Theory Group, \\ Science Park 105, 1098 XG Amsterdam, The Netherlands \\ ${ }^{d}$ Departamento de Física, Universidad de Concepción, \\ Casilla 160-C, Concepción, Chile \\ E-mail: andres.anabalon@uai.cl, B.deWit@uu.nl, juoliva@udec.cl
}

ABSTRACT: We study traversable wormhole solutions in pure gauged $N=2$ supergravity with and without electromagnetic fields, which are locally isometric under $\operatorname{SO}(2,1) \times$ $\mathrm{SO}(1,1)$. The model allows for $1 / 2$-BPS wormhole solutions whose corresponding globally defined Killing spinors are presented. A non-contractible cycle can be obtained by compactifying one of the coordinates which leaves the residual supersymmetry unaffected, although not all the isometries will remain realized globally. The wormholes connect two asymptotic, locally $\mathrm{AdS}_{4}$ regions and depend on certain electric and magnetic charge parameters and, implicitly, on the range of the compact coordinate around the throat. We provide an analysis of the boundary of the spacetime and show that it can be either disconnected or not, depending on the values of the parameters in the metric. Finally, we show how a class of these space-times avoid a topological censorship theorem.

KEYwords: Spacetime Singularities, Supergravity Models

ARXIV EPRINT: 2001.00606 


\section{Contents}

1 Introduction 1

2 The supergravity model $\quad 3$

3 Maxwell-Einstein-AdS wormholes $\quad 7$

4 Supersymmetric wormholes $\quad 10$

5 Geometric aspects of supersymmetric wormholes $\quad 14$

$\begin{array}{ll}5.1 \text { The geometry of the conformal boundary } & 15\end{array}$

$\begin{array}{lll}5.2 & \text { Geodesics and a parallel propagating singularity } & 18\end{array}$

$\begin{array}{llr}6 & \text { Topological censorship } & 20\end{array}$

$\begin{array}{llr}7 & \text { Conclusion } & 20\end{array}$

\section{Introduction}

The term "wormhole" was first introduced in a paper by Fuller and Wheeler [1], where credit was given to Weyl for the idea of having a non-simply connected space-time. Indeed, this is an essential feature of a wormhole has. It is the mathematical realization of the idea that within the same universe it is possible to travel between two points using two different paths. These ideas were thought to happen within a single universe with a single boundary. In this paper we will explicitly construct such a non-simply connected space-time with a single boundary by compactifying one non-compact coordinate of global $\mathrm{AdS}_{4}$.

We should add that the idea of a wormhole is also associated with the work by Einstein and Rosen [2], where non-singular coordinate patches of the Schwarzschild and the Reissner-Nordström black holes were studied. However, it is important to realize that the latter wormholes are very different from the one of Fuller and Wheeler, because the Einstein-Rosen bridge connects two disjoint universes that are causally disconnected. In this paper, we show that when our single boundary wormhole is extended to the nonconstant-curvature case a parallel propagating singularity develops at the boundary.

Despite these interesting features, wormholes have been widely regarded as a science fiction character. As discussed in [3, 4], this is due to the fact that the null-energy condition has to be violated at the throat of a spherically symmetric, static wormhole. ${ }^{1}$ Hence, for

\footnotetext{
${ }^{1}$ It is interesting to note that there is a widespread belief in the literature against the existence of traversable wormholes under physically sensible energy conditions. This is primarily based on the analysis of [5], where it is claimed that a four-dimensional space-time cannot have a wormhole with a minimal $\mathbb{S}^{2}$ when the null-energy condition holds. However, the wormhole studied in this paper has a minimal $\mathbb{S}^{1}$ and therefore the analysis of [5] does not apply. Indeed, the matter content that we use does satisfy the null-energy condition.
} 
asymptotically flat space-times there is not much hope for wormholes to exist in a physically sensible situation. The situation changes in asymptotically AdS space-times. When the four-dimensional space-time is Einstein, and its conformal boundary has positive scalar curvature with a space-time that is everywhere regular, then the boundary cannot have more than one connected component. If the boundary has negative scalar curvature, it is possible to construct a Euclidean wormhole by identifications in global AdS [6]. Some of these identifications have been analyzed in Euclidean AdS and several arguments have been given against the stability of these wormholes [7]. A standard one is that conformally coupled scalar fields living on a conformal boundary of negative curvature will have an action that is unbounded from below. This is indeed correct when the boundary is in the conformal class of $\mathbb{H}^{2} \times \mathbb{R}$. However, when the boundary is itself an AdS space-time this argument no longer applies for conformally coupled scalar fields, as their masses are always above the Breitenlohner-Freedman bound $[8,9]$.

This observation motivated the work in [10], where a class of geometrically non-trivial solutions was constructed where the boundary is composed of two, possibly warped, $\mathrm{AdS}_{3}$ space-times. Here we show that when the boundary is not warped $\mathrm{AdS}_{3}$, but just a locally $\mathrm{AdS}_{3}$ space-time, the proper time needed to go from one of the $\mathrm{AdS}_{3}$ components of the boundary to the other is longer through the bulk than through the boundary. This is what is called a long wormhole [11]. When the boundary has two warped $\mathrm{AdS}_{3}$ components, we shall argue that they are actually disconnected due to the existence of a parallelly propagated curvature singularity at what should be boundary of warped $\mathrm{AdS}_{3}$. However, a wormhole is not characterized exclusively by the number of boundaries it has. As discussed in [1], the wormhole can only exist provided the manifold is non-simply connected. When the noncontractible cycle has minimal length, one is dealing with a wormhole throat. As we shall see, this can already be achievable at the level of a constant curvature manifold, namely a locally $\mathrm{AdS}_{4}$ space-time. In the coordinates that we use this is implemented by requiring that one coordinate of global $\mathrm{AdS}_{4}$ to be compactified. After this identification the space-time is no longer globally $\mathrm{AdS}_{4}$ but a Lorentzian wormhole of constant curvature. The same phenomenon exists for the solutions discussed in [10], which contain non-trivial electromagnetic fields. These solutions also describe wormholes upon introducing a non-contractible cycle.

In this paper we discuss a number of new results regarding the solutions proposed in [10]. First of all we will consider these solutions in the context of $N=2$ pure gauged supergravity, which contains a non-trivial photon field. We will prove that they admit $1 / 2$ BPS solutions that respect the non-simply connected topology. The reason for the latter is that the Killing spinors do not depend on the compactified coordinate. In the constantcurvature case, half of the supersymmetries are no longer globally defined, so that only half of the Killing spinors will exist.

Finally, we discuss topological censorship, which is the claim disconnected component of the boundary can not communicate in an asymptotically locally AdS space-time, we show how our uncharged solutions do not satisfy the hypothesis of this quite general theorem $[12] .^{2}$

\footnotetext{
${ }^{2}$ We thank Juan Maldacena to bring this reference to our notice and to Nava Gaddam and Krinio Marouda for suggesting that a violation of the generic condition is the way out of the theorem.
} 
The plan of the paper is as follows. Gauged $N=2$ supergravity is introduced in section 2 , followed by a discussion of the wormhole solutions in section 3 . In section 4 we then consider the possibility of supersymmetric wormholes by proving that the integrability condition for the existence of Killing spinors is satisfied. Subsequently we present an explicit construction of the Killing spinors in section 4 and show that they are globally defined and fully compatible with the global features of the background. Some geometric aspects of supersymmetric wormholes are discussed in section 5 . When the boundary of the spacetime has two $\mathrm{AdS}_{3}$ components, we show how they can be compactified in a single $\mathbb{S}^{2} \times \mathbb{R}$, which implies that these two boundaries are connected. We compute the time that a massless particle needs to go through the bulk wormhole and verify that indeed is longer than takes a shortcut through a geodesic lying completely on the boundary. Moreover, we show that these two geodesics are not-homotopic. Hence, the wormhole agrees with the expectations of [11]. When the boundary of the space-time has two warped $\mathrm{AdS}_{3}$ components, we argue that the existence of a parallelly propagated curvature singularity imply that the boundaries are disconnected. Finally we discuss topological censorship for the uncharged solutions and point out how these results generalize to the charged case. Our conclusions are presented in section 7.

\section{The supergravity model}

In this section we present various features of pure $N=2$ supergravity with electrically charged gravitini. As is well known, supersymmetry will then imply the presence of a cosmological term whose coefficient is proportional to the square of the gravitino charge. This theory was originally constructed in terms of the physical fields $[13,14]$, whose supersymmetry transformations only close under commutation up to equations of motion. Subsequently two alternative constructions were presented based on the superconformal multiplet calculus [15-17]. The physical degrees of freedom of this theory are described by the vierbein field $e_{\mu}^{a}$, electrically charged gravitini $\psi_{\mu}$, and a photon field $A_{\mu}$. In addition we employ a spin-connection field $\omega_{\mu}^{a b}$ associated with (local) Lorentz transformations, which is not an independent field. The gravitational coupling constant has been absorbed in the fields, and the gravitino charge is equal to $q$. The gravitino fields act as the gauge fields associated with local supersymmetry. ${ }^{3}$

The $N=2$ supersymmetry transformations are described by two Majorana spinor parameters distinguished by an index $i=1,2$, and are decomposed in terms of their chiral components. The reason is that $N=2$ supersymmetry in four space-time dimensions has a chiral R-symmetry group $\mathrm{SU}(2) \times \mathrm{U}(1)$. Therefore it makes sense to consider a doublet of positive-chirality spinor parameters denoted by $\epsilon^{i}$ and a similar doublet of negativechirality parameters $\epsilon_{i}$, which each transform according under R-symmetry. As it turns out the electromagnetic gauge transformations correspond to an abelian subgroup of the

\footnotetext{
${ }^{3}$ World indices $\mu, \nu, \ldots$, and tangent-space indices $a, b, \ldots$, both run from 0 to 3 . The gamma matrices satisfy $\left\{\gamma_{a}, \gamma_{b}\right\}=2 \eta_{a b} \mathbf{1}$, where the tangent-space metric equals $\eta_{a b}=\operatorname{diag}(-1,1,1,1)$. Furthermore $\gamma^{5}=-\mathrm{i} \gamma^{0} \gamma^{1} \gamma^{2} \gamma^{3}$. In four space-time dimensions the charge-conjugation matrix $C$ is anti-symmetric and gamma matrices $\gamma_{a}$ satisfy $C \gamma_{a} C^{-1}=-\gamma_{a}{ }^{\mathrm{T}}$.
} 
$\mathrm{SU}(2) \mathrm{R}$-symmetry group. We denote the generator of this subgroup by $t_{j}^{i}$, which is thus an anti-hermitian traceless matrix. The fact that we are dealing with Majorana spinors implies that the Dirac conjugate of a chiral spinor is proportional to the anti-chiral spinor, and vice versa. For instance, the Dirac conjugate of $\epsilon^{i}$ is denoted by $\bar{\epsilon}_{i}$, where the conjugate must carry a lower $\mathrm{SU}(2)$ index, and $C \bar{\epsilon}_{i}^{\mathrm{T}}=\epsilon_{i}$, where $C$ is the charge-conjugation matrix and the superscripte $\mathrm{T}$ indicates that we have taken the transpose.

Obviously these spinorial properties are carried over to the gravitino fields, where we again distinguish two chiral doublets satisfying

$$
\gamma_{5} \psi_{\mu}^{i}=+\psi_{\mu}^{i}, \quad \gamma_{5} \psi_{\mu i}=-\psi_{\mu i} .
$$

The results given below were taken from [17], where a large class of $N=2$ theories was presented. Here we consider the following supergravity Lagrangian (up to terms quartic in the gravitini),

$$
\begin{aligned}
\mathcal{L}= & -\frac{1}{2} e R(\omega, e)-\frac{1}{8} e F(A)_{\mu \nu} F(A)^{\mu \nu} \\
& -\frac{1}{2} e\left[\bar{\psi}_{\mu}^{i} \gamma^{\mu \nu \rho} \mathcal{D}_{\nu} \psi_{\rho i}-\bar{\psi}_{\mu i} \gamma^{\mu \nu \rho} \mathcal{D}_{\nu} \psi_{\rho}^{i}\right] \\
& +\frac{1}{8} F(A)^{\rho \sigma}\left[\varepsilon_{i j} \bar{\psi}_{\mu}^{i} \gamma^{[\mu} \gamma_{\rho \sigma} \gamma^{\nu]} \psi_{\nu}^{j}+\varepsilon^{i j} \bar{\psi}_{\mu i} \gamma^{[\mu} \gamma_{\rho \sigma} \gamma^{\nu]} \psi_{\nu j}\right] \\
& +\frac{1}{2} \sqrt{2} q e\left[\varepsilon_{i k} t^{k}{ }_{j} \bar{\psi}_{\mu}^{i} \gamma^{\mu \nu} \psi_{\nu}{ }^{j}+\varepsilon^{i k} t_{k}^{j} \bar{\psi}_{\mu i} \gamma^{\mu \nu} \psi_{\nu j}\right]+6 q^{2} e,
\end{aligned}
$$

where $F(A)_{\mu \nu}=\partial_{\mu} A_{\nu}-\partial_{\nu} A_{\mu}$ and $e=\operatorname{det}\left(e_{\mu}^{a}\right)$. The derivative of the gravitino fields is covariant with respect to local Lorentz and electromagnetic gauge transformations, and reads

$$
\begin{aligned}
\mathcal{D}_{\mu} \psi_{\nu}{ }^{i} & =\left(\partial_{\mu}-\frac{1}{4} \omega_{\mu}^{a b} \gamma_{a b}\right) \psi_{\nu}^{i}-\frac{1}{2} \sqrt{2} q A_{\mu} t_{j}^{i} \psi_{\nu}{ }^{j}, \\
\mathcal{D}_{\mu} \psi_{\nu i} & =\left(\partial_{\mu}-\frac{1}{4} \omega_{\mu}^{a b} \gamma_{a b}\right) \psi_{\nu i}-\frac{1}{2} \sqrt{2} q A_{\mu} t_{i}^{j} \psi_{\nu j},
\end{aligned}
$$

where $\omega_{\mu}^{a b}$ is the spin connection whose definition will be discussed momentarily. The matrices $\frac{1}{2} \gamma_{a b}=\frac{1}{4}\left[\gamma_{a}, \gamma_{b}\right]$ are the Lorentz group generators in the spinor representation. As mentioned already, $t_{j}^{i}$ is the anti-hermitian traceless generator of the electromagnetic gauge transformations, which is an abelian subgroup of $\mathrm{SU}(2)$. It is normalized to $t_{j}^{i} t_{i}{ }^{j}=2$, where $t_{i}^{j}$ denotes the complex conjugate of $t_{j}^{i}$. This implies the convenient identities,

$$
t_{j}^{i} t_{k}{ }^{j}=\delta_{k}^{i}=-t_{j}^{i} t_{k}{ }_{k} \quad \varepsilon_{i k} t^{k}{ }_{j}=\varepsilon_{j k} t_{i}^{k}, \quad t_{i}{ }^{j} \equiv\left(t_{j}^{i}\right)^{*}=\varepsilon_{i k} \varepsilon^{j l} t^{k}{ }_{l} .
$$

These identities do not lead to a unique choice for $t_{j}^{i}$; this is consistent with the fact that the matrix can be redefined by applying a uniform chiral SU(2) field redefinition on the spinors.

The spin connection $\omega_{\mu}^{a b}$ is derived from the supercovariant torsion constraint,

$$
\mathcal{D}(\omega)_{\mu} e_{\nu}^{a}-\mathcal{D}(\omega)_{\nu} e_{\mu}^{a}=\frac{1}{2}\left[\bar{\psi}_{\mu i} \gamma^{a} \psi_{\nu}^{i}+\bar{\psi}_{\mu}^{i} \gamma^{a} \psi_{\nu i}\right]
$$

where the Lorentz covariant derivative reads $\mathcal{D}(\omega)_{\mu} e_{\nu}^{a}=\partial_{\mu} e_{\nu}{ }^{a}-\omega^{a b} e_{\nu b}$. This constraint can be solved algebraically and leads to,

$$
\omega_{\mu}^{a b}=\frac{1}{2} e_{\mu}^{c}\left(\Omega_{c}^{a b}-\Omega_{c}^{b}{ }^{a}-\Omega_{c}^{a b}\right)
$$


where the $\Omega_{a b}^{c}$ are the objects of anholonomity. The affine connection equals $\Gamma_{\mu \nu}{ }^{\rho}=$ $e_{a}^{\rho} \mathcal{D}_{\mu}(\omega) e_{\nu}^{a}$, and ensures the validity of the vielbein postulate. In the absence of torsion, where the right-hand side of (2.5) vanishes, we have

$$
\Omega_{a b}^{c}=e_{a}^{\mu} e_{b}^{\nu}\left(\partial_{\mu} e_{\nu}^{c}-\partial_{\mu} e_{\nu}^{c}\right)
$$

The corresponding expression for the affine connection is then equal to the Christoffel connection.

The curvature associated with the spin connection equals

$$
R_{\mu \nu}^{a b}(\omega)=\partial_{\mu} \omega_{\nu}^{a b}-\partial_{\nu} \omega_{\mu}^{a b}-\omega_{\mu}^{a c} \omega_{\nu c}^{b}+\omega_{\nu}^{a c} \omega_{\mu c}^{b},
$$

which satisfies the Bianchi identity $\mathcal{D}(\omega)_{[\mu} R_{\nu \rho]}{ }^{a b}(\omega)=0$. After converting the tangentspace indices in $R_{\mu \nu}^{a b}(\omega)$ to world indices, it will be equal to the Riemann tensor, up to terms quadratic in the gravitino fields that originate from the right-hand side in (2.5). Its contractions,

$$
R_{\mu}^{a}(e, \omega)=e_{b}^{\nu} R_{\mu \nu}^{a b}(\omega), \quad R(e, \omega)=e_{a}^{\mu} e_{b}^{\nu} R_{\mu \nu}^{a b}(\omega),
$$

yield the Ricci tensor and scalar, up to gravitino terms. Substituting the solution of (2.5) into $R(e, \omega)$ yields the Ricci scalar up to terms quartic in the gravitino fields.

Let us now list the supersymmetry transformation rules,

$$
\begin{aligned}
\delta e_{\mu}^{a} & =\bar{\epsilon}^{i} \gamma^{a} \psi_{\mu i}+\bar{\epsilon}_{i} \gamma^{a} \psi_{\mu}{ }^{i}, \\
\delta \psi_{\mu}^{i} & =2 \mathcal{D}_{\mu} \epsilon^{i}-\frac{1}{4} F(A)_{\rho \sigma} \gamma^{\rho \sigma} \gamma_{\mu} \varepsilon^{i j} \epsilon_{j}+\sqrt{2} q \varepsilon^{i j} t_{j}^{k} \gamma_{\mu} \epsilon_{k}, \\
\delta \psi_{\mu i} & =2 \mathcal{D}_{\mu} \epsilon_{i}-\frac{1}{4} F(A)_{\rho \sigma} \gamma^{\rho \sigma} \gamma_{\mu} \varepsilon_{i j} \epsilon^{j}+\sqrt{2} q \varepsilon_{i j} t_{k}^{j} \gamma_{\mu} \epsilon^{k}, \\
\delta A_{\mu} & =2\left(\varepsilon^{i j} \bar{\epsilon}_{i} \psi_{\mu j}+\varepsilon_{i j} \bar{\epsilon}^{i} \psi_{\mu}{ }^{j}\right),
\end{aligned}
$$

where in the gravitino transformations we suppressed terms cubic in the gravitino fields. The covariant derivatives of the supersymmetry parameters are given by

$$
\begin{aligned}
& \mathcal{D}_{\mu} \epsilon^{i}=\left(\partial_{\mu}-\frac{1}{4} \omega_{\mu}^{a b} \gamma_{a b}\right) \epsilon^{i}-\frac{1}{2} \sqrt{2} q A_{\mu} t^{i}{ }_{j} \epsilon^{j}, \\
& \mathcal{D}_{\mu} \epsilon_{i}=\left(\partial_{\mu}-\frac{1}{4} \omega_{\mu}^{a b} \gamma_{a b}\right) \epsilon_{i}-\frac{1}{2} \sqrt{2} q A_{\mu} t_{i}{ }^{j} \epsilon_{j} .
\end{aligned}
$$

The Lagrangian (2.2) is invariant under space-time diffeomorphisms, supersymmetry, local Lorentz transformations, and electromagnetic gauge transformations, whose infinitesimal transformations will close under commutation. Of particular interest is the commutator of two supersymmetry transformations, which closes into the diffeomorphism with parameter $\xi^{\mu}$, the local Lorentz transformations and the electromagnetic gauge transformations, but only modulo the gravitino field equations,

$$
\left[\delta\left(\epsilon_{1}\right), \delta\left(\epsilon_{2}\right)\right]=\xi^{\mu} \hat{D}_{\mu}+\delta_{\mathrm{L}}(\varepsilon)+\delta(\Lambda)
$$


where the derivative is fully covariant with respect to all the symmetries. This implies that there is a contribution from $\xi^{\mu}$ times each of the connections that contribute. The explicit variations are simply additional and they do not involve the connections. The parameters of the various infinitesimal transformations on the right-hand side are given by

$$
\begin{aligned}
\xi^{\mu} & =2 \bar{\epsilon}_{2}{ }^{i} \gamma^{\mu} \epsilon_{1 i}+\text { h.c. } \\
\varepsilon^{a b} & =\varepsilon_{i j} \bar{\epsilon}_{1}{ }^{i} \epsilon_{2}{ }^{j} F^{a b+}+\text { h.c. }, \\
\Lambda & =4 \varepsilon_{i j} \bar{\epsilon}_{2}{ }^{i} \epsilon_{1}^{j}+\text { h.c. }
\end{aligned}
$$

where the first term proportional to $\xi^{\mu}$ denotes a supercovariant translation, i.e. a general coordinate transformation with parameter $\xi^{\mu}$, suitably combined with field-dependent gauge transformations so that the result is supercovariant.

We will be interested in solutions that have full or partial supersymmetry. The fully supersymmetric solution is well known and we will briefly refer to it at the end of this section. There exist many solutions with partial supersymmetry. Well-known examples are, for instance, the extremal Reissner-Nordström black holes solutions, which are invariant under half the supersymmetries [18]. However, the main objective of this paper is to analyze the possible supersymmetry of wormhole solutions belonging to the class constructed in [10].

When a bosonic field configuration is fully or partially supersymmetric, it implies that all or some of the supersymmetry transformations of the fermions are vanishing. The transformations that vanish are characterized by certain spinorial parameters that are known as generalized Killing spinors. The only spinors that we are dealing with in this particular case are the gravitini, so we have to simply analyse their supersymmetry transformation, which amounts to deriving possible solutions for $\epsilon^{i}$ and $\epsilon_{i}$ of the equations,

$$
\begin{aligned}
& 2 \mathcal{D}_{\mu} \epsilon^{i}-\frac{1}{4} F(A)_{\rho \sigma} \gamma^{\rho \sigma} \gamma_{\mu} \varepsilon^{i j} \epsilon_{j}+\sqrt{2} q \varepsilon^{i j} t_{j}{ }^{k} \gamma_{\mu} \epsilon_{k}=0, \\
& 2 \mathcal{D}_{\mu} \epsilon_{i}-\frac{1}{4} F(A)_{\rho \sigma} \gamma^{\rho \sigma} \gamma_{\mu} \varepsilon_{i j} \epsilon^{j}+\sqrt{2} q \varepsilon_{i j} t^{j}{ }_{k} \gamma_{\mu} \epsilon^{k}=0 .
\end{aligned}
$$

It is convenient to first consider an integrability condition for these differential equations, which follows by applying a second derivative $\mathcal{D}_{\nu}$ and anti-symmetrizing over the indices $\mu$ and $\nu$. The resulting equations take the following form,

$$
\begin{aligned}
\Xi_{\mu \nu}{ }^{i} \equiv & \mathcal{D}_{\mu} \delta \psi_{\nu}^{i}-\mathcal{D}_{\nu} \delta \psi_{\mu}^{i}=\left[R(\omega)_{\mu \nu}^{a b} \gamma_{a b}-4 q^{2} \gamma_{\mu \nu}+\frac{1}{8} F_{\rho \sigma} F_{\lambda \tau} \gamma^{\rho \sigma} \gamma_{[\mu} \gamma^{\lambda \tau} \gamma_{\nu]}\right] \epsilon^{i} \\
& +\frac{1}{2} \sqrt{2} q\left[4 F_{\mu \nu}-F_{\rho \sigma} \gamma^{\rho \sigma} \gamma_{\mu \nu}-\gamma_{[\mu} F_{\rho \sigma} \gamma^{\rho \sigma} \gamma_{\nu]}\right] t_{j}^{i} \epsilon^{j}+\left(\nabla_{[\mu} F_{\rho \sigma}\right) \gamma^{\rho \sigma} \gamma_{\nu]} \varepsilon^{i j} \epsilon_{j}=0, \\
\Xi_{\mu \nu i} \equiv & \mathcal{D}_{\mu} \delta \psi_{\nu i}-\mathcal{D}_{\nu} \delta \psi_{\mu i}=\left[R(\omega)_{\mu \nu}^{a b} \gamma_{a b}-4 q^{2} \gamma_{\mu \nu}+\frac{1}{8} F_{\rho \sigma} F_{\lambda \tau} \gamma^{\rho \sigma} \gamma_{[\mu} \gamma^{\lambda \tau} \gamma_{\nu]}\right] \epsilon_{i} \\
& +\frac{1}{2} \sqrt{2} q\left[4 F_{\mu \nu}-F_{\rho \sigma} \gamma^{\rho \sigma} \gamma_{\mu \nu}-\gamma_{[\mu} F_{\rho \sigma} \gamma^{\rho \sigma} \gamma_{\nu]}\right] t_{i}^{j} \epsilon_{j}+\left(\nabla_{[\mu} F_{\rho \sigma}\right) \gamma^{\rho \sigma} \gamma_{\nu]} \varepsilon_{i j} \epsilon^{j}=0,
\end{aligned}
$$

where the covariant derivative $\nabla_{\mu}$ contains only the Christoffel connection. 
To analyze the above equations it is convenient to switch from two Majorana spinors to a single Dirac spinor. To do so, one first chooses, without loss of generality, the charge matrix $t^{i}{ }_{j}$ to be equal to $\operatorname{diag}(\mathrm{i},-\mathrm{i})$. Subsequently one defines

$$
\chi \equiv \epsilon^{1}+\epsilon_{2}, \quad \Xi_{\mu \nu} \chi \equiv \Xi_{\mu \nu}^{1}+\Xi_{\mu \nu 2} .
$$

Now $\chi$ is no longer a Majorana spinor, because under charge conjugations it will lead to another independent spinor $\epsilon_{1}+\epsilon^{2}$. Since the two spinors are related by charge conjugation, it suffices to only consider the quantities $\Xi_{\mu \nu}$, which constitute six different $4 \times 4$ matrices acting on the 4-component Dirac spinor $\chi$, defined by (2.16).

With these redefinitions the Killing spinor equations (2.14) and the integrability condition (2.15) reads as follows,

$$
\begin{aligned}
2 \mathcal{D}_{\mu} \chi+ & \frac{1}{4} F(A)_{\rho \sigma} \gamma^{\rho \sigma} \gamma_{\mu} \gamma^{5} \chi-\sqrt{2} \mathrm{i} q \gamma_{\mu} \gamma^{5} \chi=0 \\
\Xi_{\mu \nu} \chi= & {\left[R(\omega)_{\mu \nu}^{a b} \gamma_{a b}-4 q^{2} \gamma_{\mu \nu}+\frac{1}{8} F_{\rho \sigma} F_{\lambda \tau} \gamma^{\rho \sigma} \gamma_{[\mu} \gamma^{\lambda \tau} \gamma_{\nu]}\right] \chi } \\
& +\frac{1}{2} \sqrt{2} \mathrm{i} q\left[4 F_{\mu \nu}-F_{\rho \sigma} \gamma^{\rho \sigma} \gamma_{\mu \nu}-\gamma_{[\mu} F_{\rho \sigma} \gamma^{\rho \sigma} \gamma_{\nu]}\right] \chi-\left(\nabla_{[\mu} F_{\rho \sigma}\right) \gamma^{\rho \sigma} \gamma_{\nu]} \gamma^{5} \chi=0
\end{aligned}
$$

The covariant derivative of $\chi$ follows from (2.11),

$$
\mathcal{D}_{\mu} \chi=\left(\partial_{\mu}-\frac{1}{4} \omega_{\mu}^{a b} \gamma_{a b}-\frac{1}{2} \sqrt{2} \mathrm{i} q A_{\mu}\right) \chi .
$$

We recall that all fermionic fields have been suppressed on the right-hand side of the equations (2.14) and (2.18), because we will be dealing with purely bosonic backgrounds when exploring the possible supersymmetry of wormhole solutions.

The maximally supersymmetric solution has vanishing $A_{\mu}$, so that the integrability relation then takes the form $R(\omega)_{\mu \nu}^{a b}=4 q^{2} e_{\mu}^{[a} e_{\nu}^{b]}$. This equation implies that the supersymmetric field configuration is just an anti-de Sitter space-time with AdS radius $\ell$ given by

$$
\ell^{-1}=\sqrt{2}|q|
$$

In the following sections we will consider a class of wormhole solutions that can be partially supersymmetric. Their possible supersymmetry will be investigated by analyzing the equations (2.17).

\section{Maxwell-Einstein-AdS wormholes}

Following [10], we consider a class of four-dimensional space-time metrics expressed into two different functions, $f(r)$ and $h(r)$,

$$
d s^{2}=\frac{4 \ell^{4} d r^{2}}{\sigma^{2} f(r)}+h(r)\left[-\cosh ^{2} \theta d t^{2}+d \theta^{2}\right]+f(r)(d u+\sinh \theta d t)^{2},
$$


where $\ell$ denotes the AdS radius. When considering supersymmetry we will also need a corresponding set of vierbeine, for which we make the following choice,

$$
\begin{aligned}
e^{0} & =\sqrt{h(r)} \cosh \theta d t, \\
e^{1} & =\frac{1}{\sigma q^{2} \sqrt{f(r)}} d r, \\
e^{2} & =\sqrt{h(r)} d \theta, \\
e^{3} & =\sqrt{f(r)}(d u+\sinh \theta d t) .
\end{aligned}
$$

For

$$
\sigma=4, \quad f(r)=h(r)=\frac{1}{4} \ell^{2}\left(r^{2}+1\right),
$$

this defines a global $\mathrm{AdS}_{4}$ space-time. Its topology is trivial because the coordinates cover the full $\mathbb{R}^{4}$.

However, it is possible to impose identifications on surfaces that are orthogonal to $\partial_{r}$ so that one obtains a constant curvature wormhole. In this case the space-time is only locally $\mathrm{AdS}_{4}$ and has two conformal boundaries located at $r= \pm \infty$. The relevant identification in the Lorentzian case is $u \sim u+a$, which for constant $r$ yields the threedimensional Cousaert-Henneaux space-time [19]. This identification obviously introduces a non-contractible cycle in space-time. In the case at hand, the location of the throat is at $r=0$, when the non-contractible circle has minimal (geodesic) length. The perimeter of the throat given by $a$ is an extra parameter of the metric that is encoded in the range of the compact coordinate $u \in[0, a]$. This is not sufficient to prove that this geometry defines a traversable wormhole. For that one needs to send information from one side of the throat to the other, and one has to check that there are no closed time-like curves. The later was addressed in [10] for the uncharged case and the same argument applies here; the former will be studied in section 5 below.

Since this field configuration is a solution of the Einstein-Maxwell system with a cosmological term, it can also be a solution of pure $N=2$ supergravity, which means that it is a solution of its bosonic field equations that follow from the Lagrangian (2.2). These combined field equations that it satisfies will therefore take the form

$$
\begin{aligned}
& \partial_{\mu}\left(e F^{\mu \nu}\right)=0, \\
& R_{\mu \nu}-\frac{1}{2} g_{\mu \nu} R+\frac{1}{2}\left[F_{\mu \rho} F_{\nu}{ }^{\rho}-\frac{1}{4} g_{\mu \nu} F_{\rho \sigma} F^{\rho \sigma}\right]+6 q^{2} g_{\mu \nu}=0,
\end{aligned}
$$

where $\ell^{-1}=\sqrt{2}|q|$ and $F_{\mu \nu}=0$.

Let us now move to a more complicated metric where the functions $f(r)$ and $h(r)$ are equal to

$$
f(r)=\frac{2}{q^{2} \sigma^{2}} \frac{r^{4}+(6-\sigma) r^{2}+m r+\sigma-3}{r^{2}+1}-\frac{Q^{2}+P^{2}}{r^{2}+1}, \quad h(r)=\frac{1}{2 q^{2} \sigma}\left(r^{2}+1\right),
$$

and construct a corresponding solution of the above equations. Here $Q$ and $P$ are electric and magnetic charge parameters that will determine the physical charges (whose definition requires to properly account for wormhole topology) and the corresponding electric 
and magnetic fields of the solution. These charges are induced because the second field equation (3.4) requires the presence of electric and magnetic fields, which will be given momentarily. Note, however, that we still retain the homogeneous Maxwell equations, because the only charged sources are the gravitini, which are not included in the bosonic background solution. In addition the metric depends on two integration constants denoted by $m$ and $\sigma$. The parameter $m$ is proportional to the mass of the space-time, while $\sigma$ is related to the warping of the asymptotic region. More details can be found in [10].

The solution of (3.4) for the vector potential is given by

$$
A=\Phi(r)(d u+\sinh \theta d t)
$$

with $\Phi(r)$ equal to

$$
\Phi(r)=\frac{2 Q r+P\left(1-r^{2}\right)}{r^{2}+1}
$$

It turns out that (3.6) is invariant under the isometries given below in (3.11). Obviously the vector potential $A_{\mu}$ describes an electric and a magnetic field component. Its field strength in the adopted coordinate system is equal to

$$
\begin{aligned}
F_{r u} & =\frac{2\left(1-r^{2}\right) Q-4 r P}{\left(r^{2}+1\right)^{2}}, \\
F_{r t} & =\frac{2\left(1-r^{2}\right) Q-4 r P}{\left(r^{2}+1\right)^{2}} \sinh \theta, \\
F_{\theta t} & =\frac{2 Q r+P\left(1-r^{2}\right)}{r^{2}+1} \cosh \theta .
\end{aligned}
$$

The possible existence of a non-contractible cycle requires that $f(r)$ must be positive everywhere. ${ }^{4}$ Asymptotically, for $r= \pm \infty$ the space-time is locally $\mathrm{AdS}_{4}$ with the following fall-off for the curvature tensor,

$$
R(\omega)_{\mu \nu}^{a b}=\left[2 \ell^{-2}+\mathcal{O}\left(r^{-2}\right)\right] e_{\mu}^{[a} e_{\nu}^{b]} .
$$

The bosonic field configuration associated with global $\mathrm{AdS}_{4}$ is invariant under the isometry group $\mathrm{SO}(3,2)$. This group is broken for the deformed functions $f(r)$ and $h(r)$ specified in (3.5) and the electromagnetic fields (3.8) to a subgroup generated by the fol-

\footnotetext{
${ }^{4}$ A straightforward analysis shows that $f(r)$ never vanishes provided

$$
\begin{aligned}
& X=3\left(Q^{2}+P^{2}\right) \ell^{-2} \leq 1, \quad \frac{12+12 \sqrt{1-X}}{1+X+\sqrt{1-X}}>\sigma>\frac{12-6 \sqrt{1-X}}{1+X+\sqrt{1-X}} \\
& |m|<\frac{\sqrt{2}}{3 \sqrt{3}} \frac{\sigma(6-\sigma) \sqrt{1-X}+24 \sigma-\sigma^{2}(1+X)-72}{\sqrt{\sigma(1+\sqrt{1-X})-6}}
\end{aligned}
$$
}

For these ranges of the parameters, the metric functions are everywhere positive and regular and a nontrivial wormhole space-time will exist. 
lowing four Killing vectors,

$$
\begin{aligned}
& \xi_{[1]}=\partial_{t}, \\
& \xi_{[2]}=\sin t \partial_{\theta}+\tanh \theta \cos t \partial_{t}+\frac{\cos t}{\cosh \theta} \partial_{u}, \\
& \xi_{[3]}=\cos t \partial_{\theta}-\tanh \theta \sin t \partial_{t}-\frac{\sin t}{\cosh \theta} \partial_{u}, \\
& \xi_{[4]}=\partial_{u} .
\end{aligned}
$$

We note that when $u$ is compact the $\mathrm{SO}(3,2)$ isometries of global $\mathrm{AdS}_{4}$ are also broken to these four Killing vectors. The first three Killing vectors generate the group $\mathrm{SO}(2,1)$, while the fourth isometry is abelian and commutes with the first three. Not surprisingly, the two functions given in (3.5) depend only on $r$ and are therefore invariant under the four isometries. Our solution can be seen as a deformation of $\mathrm{AdS}_{3}$ embedded in a fourdimensional space. The deformation by the function $f(r)$ breaks the $\mathrm{SO}(2,2) \cong \mathrm{SO}(2,1) \times$ $\mathrm{SO}(2,1)$ isometries to its subgroup $\mathrm{SO}(2,1) \times \mathrm{SO}(1,1)$.

We also calculate $\mathcal{L}_{\xi} e_{\mu}^{a}=\xi^{\nu} \partial_{\nu} e_{\mu}^{a}+\partial_{\mu} \xi^{\nu} e_{\nu}^{a}$ for each of the Killing vectors. As it turns out $\mathcal{L}_{\xi} e_{\mu}^{a}$ vanishes on all the vierbeine for the $\xi_{[1]}$ and $\xi_{[4]}$, while the non-trivial action of the other Killing vectors on the vierbeine yields

$$
\begin{array}{ll}
\mathcal{L}_{\xi_{[2]}} e^{0}=\frac{\cos t}{\cosh \theta} e^{2}, & \mathcal{L}_{\xi_{[3]}} e^{0}=-\frac{\sin t}{\cosh \theta} e^{2}, \\
\mathcal{L}_{\xi_{[2]}} e^{2}=\frac{\cos t}{\cosh \theta} e^{0}, & \mathcal{L}_{\xi_{[3]}} e^{2}=-\frac{\sin t}{\cosh \theta} e^{0} .
\end{array}
$$

Hence the vierbeine are not invariant under the diffeomorphisms generated by the Killing vectors, but they are invariant under these diffeomorphisms when accompanied by tangentspace transformations that are opposite to the ones indicated above. On spinors these tangent transformations will take the form

$$
\delta_{[2]} \psi=-\frac{\cos t}{2 \cosh \theta} \gamma^{0} \gamma^{2} \psi, \quad \delta_{[3]} \psi=\frac{\sin t}{2 \cosh \theta} \gamma^{0} \gamma^{2} \psi .
$$

We will return to these compensating tangent-space transformation at the end of sections 4, where we will discuss the corresponding invariances of the Killing spinors. Note that the transformations $\xi_{[1]}$ and $\xi_{[4]}$ do not involve any compensating tangent-space transformations.

\section{Supersymmetric wormholes}

To investigate whether the wormhole solutions can be supersymmetric, one may first consider the integrability for the complex Killing spinors $\chi$, which was presented in (2.17). In the actual calculations we use the following representation for the gamma matrices,

$$
\gamma^{0}=-\mathrm{i}\left(\begin{array}{cc}
0 & \sigma_{2} \\
\sigma_{2} & 0
\end{array}\right) \quad \gamma^{1}=-\left(\begin{array}{cc}
\sigma_{3} & 0 \\
0 & \sigma_{3}
\end{array}\right), \quad \gamma^{2}=\mathrm{i}\left(\begin{array}{cc}
0 & -\sigma_{2} \\
\sigma_{2} & 0
\end{array}\right), \quad \gamma^{3}=\left(\begin{array}{cc}
\sigma_{1} & 0 \\
0 & \sigma_{1}
\end{array}\right),
$$

where we remind the reader of the definition $\gamma^{5}=-\mathrm{i} \gamma^{0} \gamma^{1} \gamma^{2} \gamma^{3}=\operatorname{diag}\left(\sigma_{2},-\sigma_{2}\right){ }^{5}$

\footnotetext{
${ }^{5}$ With these gamma matrices we can choose the charge conjugation matrix as $S=S^{-1}=-S^{\mathrm{T}}$, so that the charge conjugate of a spinor $\psi$ is equal to $S \bar{\psi}^{\mathrm{T}}=\psi^{*}$.
} 
A necessary condition for the existence of non-trivial Killing spinors is that the determinant of each of the six $4 \times 4$ matrices $\Xi_{\mu \nu}$ defined in (2.16) must vanish. As it turns out all six determinants take the form of a constant times $\left(r^{2}+1\right)^{-6}$ times a function $Z(r)$. This function also depends on the charges and the integration constants $\sigma$ and $m$ in the metric based on (3.5) so the condition for supersymmetry is that $Z(r)$ must vanish. Explicit calculation shows that the function $Z(r)$ has the following form,

$$
Z(r)=Z_{2} r^{2}+Z_{1} r+Z_{0},
$$

where $Z_{2}, Z_{1}$ and $Z_{0}$ are fairly complicated expressions that contain the charges and integration constants. However, the integrability condition should hold for any value of the radial coordinate $r$. Therefore one concludes that $Z_{2}, Z_{1}$ and $Z_{0}$ should separately vanish. For $Z_{2}$ this leads to the equation,

$$
Z_{2}=\frac{\sigma^{2}}{q^{4}}(m P+8 Q-2 Q \sigma)=0 .
$$

Since the metric is singular when $\sigma$ vanishes, we conclude that

$$
m=\frac{2 Q}{P}(\sigma-4)
$$

When this equation is satisfied then $Z_{1}$ turns out to vanish identically. Hence the only remaining condition follows from requiring that $Z_{0}$ must vanish,

$$
Z_{0}=\frac{\left(P^{2}+Q^{2}\right)^{2}}{2 P^{4} q^{6}}\left(2 q^{2} \sigma^{2} P^{2}+(\sigma-4)^{2}\right)\left(-2(\sigma-4)+P^{2} \sigma^{2} q^{2}\right)^{2}=0,
$$

where we made again use of equation (4.4). Combining the above results one obtains the conditions

$$
P=\frac{1}{|q| \sigma} \sqrt{2(\sigma-4)}, \quad m=|q| \sigma Q \sqrt{2(\sigma-4)} .
$$

Supersymmetry thus implies $\sigma>4$ which is the same result that was found in [10] by requiring holographic stability. ${ }^{6}$

Now that we have solved the integrability condition for the existence of Killing spinors, let us proceed to an explicit determination of these spinors. To appreciate the possible relevance of the identification $u \sim u+a$ for supersymmetry, we determine the possible Killing spinors explicitly. To solve the Killing spinor we use the Dirac spinor $\chi$ defined in (2.16). The Killing spinor equations for $\chi$ was already given in (2.14). Substituting the expression for the bosonic covariant derivative, it reads

$$
\left[\partial_{\mu}-\frac{1}{4} \omega_{\mu}^{a b} \gamma_{a b}-\frac{1}{2} \sqrt{2} \mathrm{i} q A_{\mu}+\frac{1}{8} F_{\rho \sigma} \gamma^{\rho \sigma} \gamma_{\mu} \gamma^{5}-\frac{1}{2} \sqrt{2} \mathrm{i} q \gamma_{\mu} \gamma^{5}\right] \chi=0 .
$$

\footnotetext{
${ }^{6}$ It is possible to define $\sigma$ in terms of the charge parameters $Q$ and $P$, but there are two solutions:

$$
\sigma_{ \pm}=\frac{1}{q^{2} P^{2}}\left(1 \pm \sqrt{1-8 q^{2} P^{2}}\right)
$$
}


It is useful to first study the Killing spinors of global $\mathrm{AdS}_{4}$ in terms of the coordinates used throughout this paper and to observe the effect of having a compact $u$-coordinate on supersymmetry. We suppress for the moment the presence of $A_{\mu}$ and $F_{\mu \nu}$ in (4.7). In this way we obtain the following four real Killing spinors,

$$
\begin{gathered}
\chi_{1}^{\mathrm{AdS}}=\left(\begin{array}{c}
\sqrt{1+\sqrt{r^{2}+1}}[\cosh \theta / 2 \cos t / 2-\sinh \theta / 2 \sin t / 2] \\
-\sqrt{-1+\sqrt{r^{2}+1}}[\cosh \theta / 2 \cos t / 2-\sinh \theta / 2 \sin t / 2] \\
\sqrt{1+\sqrt{r^{2}+1}}[\cosh \theta / 2 \sin t / 2-\sinh \theta / 2 \cos t / 2] \\
\sqrt{-1+\sqrt{r^{2}+1}}[\cosh \theta / 2 \sin t / 2-\sinh \theta / 2 \cos t / 2]
\end{array}\right), \\
\chi_{2}^{\mathrm{AdS}}=\left(\begin{array}{c}
-\sqrt{1+\sqrt{r^{2}+1}}[\sinh \theta / 2 \cos t / 2+\cosh \theta / 2 \sin t / 2] \\
\sqrt{-1+\sqrt{r^{2}+1}}[\sinh \theta / 2 \cos t / 2+\cosh \theta / 2 \sin t / 2] \\
\sqrt{1+\sqrt{r^{2}+1}}[\sinh \theta / 2 \sin t / 2+\cosh \theta / 2 \cos t / 2] \\
\sqrt{-1+\sqrt{1+r^{2}}}[\sinh \theta / 2 \sin t / 2+\cosh \theta / 2 \cos t / 2]
\end{array}\right), \\
\left.\chi_{3}^{\mathrm{AdS}}=e^{u / 2\left(\begin{array}{c}
\sqrt{-1+\sqrt{r^{2}+1}} \\
-\sqrt{1+\sqrt{r^{2}+1}} \\
0 \\
0
\end{array}\right)} \begin{array}{c}
0 \\
{ }_{4}^{\mathrm{AdS}}=e^{-u / 2} \\
\sqrt{-1+\sqrt{r^{2}+1}} \\
\sqrt{1+\sqrt{r^{2}+1}}
\end{array}\right) .
\end{gathered}
$$

However, we have to remember that we are constructing representations for complex Killing spinors, so that the above spinors can be multiplied by arbitrary complex normalization factors. Hence we are dealing with eight independent Killing spinors, which will indeed provide a basis for full $N=2$ supersymmetry, as is expected for a global $\mathrm{AdS}_{4}$ space-time.

A noteworthy feature in the context of the present paper is that the last two Dirac spinors, $\chi_{3}^{\mathrm{AdS}}$ and $\chi_{4}^{\mathrm{AdS}}$, are incompatible with a periodic coordinate $u$. Therefore, when dealing with a non-contractible cycle $u \sim u+a$, half of the Killing spinors will no longer be globally defined, so that this particular field configuration must be regarded as a $1 / 2$-BPS solution. At the same time, the equations of motion will still be locally satisfied.

At this point one can invoke the supersymmetry algebra given by (2.12), which relates the commutator of two supersymmetry transformations to the bosonic symmetries of the model. When choosing supersymmetry parameters expressed in terms of linear combinations of the Killing spinors $\epsilon^{i}$, one obtains all the bosonic transformations that should be compatible with the supersymmetric background, and in particular one would obtain the Killing vectors of $\mathrm{AdS}_{4}$. However, when the Killing spinors are not all globally defined, then some of the Killing vectors of the space-time will not be globally defined either.

Let us now continue and derive the Killing spinors for the non-constant curvature wormhole with non-trivial electromagnetic fields. A lengthy analysis shows that there exist only two Dirac, Killing spinors, so that the number of Killing spinors is reduced to one half. Furthermore, these spinors do no longer depend on the coordinate $u$, so that they are globally defined. We will give the explicit expressions momentarily. It turns out 
that the first and the second component of these spinors differ by an overall function $G(r)$, whereas the third and the fourth component differ by an overall function $\bar{G}(r)$ that equals the complex conjugate of $G(r)$. This function $G(r)$ is quite complicated and takes the following form,

$$
G(r)=\frac{-1}{q^{2} \sigma h(r)} \frac{[\sqrt{f(r)}-2 \sqrt{2} q h(r)][\sqrt{f(r)}-\mathrm{i} \Phi(r)]}{f^{\prime}(r)+\mathrm{i} \sqrt{f(r)} \Phi^{\prime}(r)} .
$$

The two Dirac Killing spinors now take the form,

$$
\begin{gathered}
\chi_{1}{ }^{\mathrm{WH}}=\alpha(r)\left(\begin{array}{c}
e^{\mathrm{i} \beta(r)}[\cosh \theta / 2 \cos t / 2-\sinh \theta / 2 \sin t / 2] \\
-e^{\mathrm{i} \beta(r)} G(r)[\cosh \theta / 2 \cos t / 2-\sinh \theta / 2 \sin t / 2] \\
e^{-\mathrm{i} \beta(r)}[\cosh \theta / 2 \sin t / 2-\sinh \theta / 2 \cos t / 2] \\
e^{-\mathrm{i} \beta(r)} \bar{G}(r)[\cosh \theta / 2 \sin t / 2-\sinh \theta / 2 \cos t / 2]
\end{array}\right), \\
\chi_{2}{ }^{\mathrm{WH}}=\alpha(r)\left(\begin{array}{c}
-e^{\mathrm{i} \beta(r)}[\sinh \theta / 2 \cos t / 2+\cosh \theta / 2 \sin t / 2] \\
e^{\mathrm{i} \beta(r)} G(r)[\sinh \theta / 2 \cos t / 2+\cosh \theta / 2 \sin t / 2] \\
e^{-\mathrm{i} \beta(r)}[\sinh \theta / 2 \sin t / 2+\cosh \theta / 2 \cos t / 2] \\
e^{-\mathrm{i} \beta(r)} \bar{G}(r)[\sinh \theta / 2 \sin t / 2+\cosh \theta / 2 \cos t / 2]
\end{array}\right),
\end{gathered}
$$

where

$$
\begin{aligned}
\alpha(r) & =\frac{h^{1 / 4}(r)}{\sqrt{1+|G(r)|^{2}}}, \\
e^{2 \mathrm{i} \beta(r)} & =\left(1+\frac{1}{2} \mathrm{i} \sqrt{\sigma-4}\right) \sqrt{\frac{f(r)}{h(r)}} \frac{1+|G(r)|^{2}}{1+G(r)^{2}} .
\end{aligned}
$$

Therefore we find that there are two independent Dirac Killing spinors (which in this case are actually complex). This solution is therefore $1 / 2$-BPS. As before we can invoke the supersymmetry algebra, and verify that one reproduces the Killing vectors (3.11), which will be globally defined. All this provides a non-trivial check of the correctness of our results.

We can also determine how these Killing spinors transform under the symmetries of the bosonic field configuration. As explained at the end of section 4, these symmetries take the form of a linear combination of the isometries (3.11) and certain tangent-space transformations that act on spinors according to (3.13). The Killing spinors thus transform under both transformations. As it turns out, the tangent space transformation will cancel in this linear combination, and we are left with the following transformations,

$$
\begin{aligned}
& \delta_{[1]} \chi^{\mathrm{WH}}=\frac{1}{2}\left(\begin{array}{cc}
0 & 1 \\
-1 & 0
\end{array}\right) \chi^{\mathrm{WH}}, \\
& \delta_{[2]} \chi^{\mathrm{WH}}=\frac{1}{2}\left(\begin{array}{cc}
-1 & 0 \\
0 & 1
\end{array}\right) \chi^{\mathrm{WH}}, \\
& \delta_{[3]} \chi^{\mathrm{WH}}=-\frac{1}{2}\left(\begin{array}{ll}
0 & 1 \\
1 & 0
\end{array}\right) \chi^{\mathrm{WH}}, \\
& \delta_{[4]} \chi^{\mathrm{WH}}=0
\end{aligned}
$$




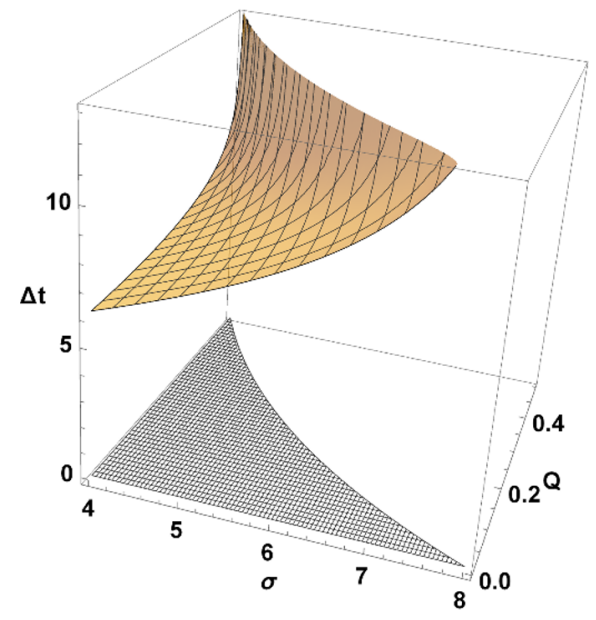

Figure 1. Crossing time $\Delta t$ for a photon as a function of the charge $Q$ and the parameter $\sigma$. The region where the metric is regular and the wormhole is BPS corresponds to the shaded area in the lower plane. This restriction originates from the bounds given in (3.9) and the BPS conditions (4.6). The crossing time remains finite, and starts growing as one approaches the upper bound on $Q$.

where

$$
\chi^{\mathrm{WH}}=\left(\begin{array}{l}
\chi_{1}{ }^{\mathrm{WH}} \\
\chi_{2}{ }^{\mathrm{WH}}
\end{array}\right) .
$$

Obviously the Killing spinors thus transform according the two-dimensional representation of $\mathrm{SO}(2,1)$.

\section{Geometric aspects of supersymmetric wormholes}

In the previous section we proved the existence of $1 / 2$-BPS wormhole solutions in $N=2$ supergravity. Now we turn to a discussion of the geometric properties of these space-times.

The throat of the supersymmetric wormhole is located at the minimum of the volume of the $t, r=$ constant surfaces. This is at the minimum of the function $f(r) \cdot h(r)$. A plot with the time it takes for a photon to cross the whole space-time, as seen by a geodesic observer located at $r=t=\theta=0$ and constant $u$, is shown in figure 1 .

An important remark is now in order. The vector $\partial_{t}$, which is asymptotically timelike for $\sigma \geq 4$ may become space-like in the interior of the wormhole when the following inequality holds,

$$
f(r) \sinh ^{2} \theta-h(r) \cosh ^{2} \theta>0 .
$$

This would lead to an ergoregion, as happens in [20], and tends to be in contradiction with supersymmetry [21]. However, from the supersymmetry conditions (4.6) one can show in a straight-forwarded manner that the inequality (5.1) cannot be fulfilled, so that the asymptotically timelike Killing vector $\partial_{t}$ is actually timelike everywhere in the interior of the BPS wormhole geometry. 



Figure 2. Embedding of the charged supersymmetric wormhole with $Q=10^{-1}$ and $\sigma=5$ (left panel) and $\sigma=6$ (right panel).

The induced metric on the surfaces at constant $t$ and $\theta$ is equal to

$$
d s^{2}=\frac{d r^{2}}{q^{4} \sigma^{2} f(r)}+f(r) d u^{2}=d \rho^{2}+R^{2}(\rho) d u^{2}
$$

where the second equation is obtained by going to the proper radial coordinate. The function $R(\rho)$ defines the radius of the circles parameterized by the compact coordinate $u$. As $r \rightarrow \pm \infty$ one has $\rho \rightarrow \pm \infty$ and $R(\rho) \sim e^{ \pm \rho}$, as expected due to the locally AdS asymptotics. The coordinate $\rho$ is such that $r=0$ implies $\rho=0$. Figure 2 shows the plot of the radial function $R(\rho)$ as a function of $\rho$. The latter runs radially on the wormhole geometry and measures the proper radial distance from the throat.

\subsection{The geometry of the conformal boundary}

Let us pass to study the asymptotic region of the space-time. To this end, it is enlightening to understand first pure $\mathrm{AdS}_{4}$ space-time. We would like to clarify the relation between the global coordinate system we use in this article and the standard global coordinate system where the space-time is foliated by spheres. AdS is the Lorentzian hyperboloid, where the AdS radius is set to one, $\ell=1$,

$$
-X_{0}^{2}-X_{1}^{2}+X_{2}^{2}+X_{3}^{2}+X_{4}^{2}=-1 .
$$

This constraint is solved by the global parametrization,

$$
\begin{array}{ll}
X_{0}^{S^{2}}=\sqrt{\rho^{2}+1} \sin \tau, & X_{1}^{S^{2}}=\sqrt{\rho^{2}+1} \cos \tau, \\
X_{2}^{S^{2}}=\rho \sqrt{1-y^{2}} \cos \phi, & X_{3}^{S^{2}}=\rho \sqrt{1-y^{2}} \sin \phi, \\
X_{4}^{S^{2}}=\rho y, &
\end{array}
$$

we shall call this the sphere foliation. It has the (universal covering) metric

$$
d s_{S^{2}}^{2}=-\left(1+\rho^{2}\right) d \tau^{2}+\frac{d \rho^{2}}{1+\rho^{2}}+\rho^{2}\left(\frac{d y^{2}}{1-y^{2}}+\left(1-y^{2}\right) d \phi^{2}\right)
$$


where $y \in[-1,1], \phi \in[0,2 \pi], \tau \in[-\infty, \infty]$ and $\rho \in[0, \infty]$. Elsewhere in this paper we have used another global parametrization,

$$
\begin{aligned}
& X_{0}^{\mathrm{AdS}_{3}}=[\sin t / 2 \sinh u / 2 \sinh \theta / 2+\cos t / 2 \cosh u / 2 \cosh \theta / 2] \sqrt{r^{2}+1} \\
& X_{1}^{\mathrm{AdS}_{3}}=[\sin t / 2 \cosh u / 2 \cosh \theta / 2-\cos t / 2 \sinh u / 2 \sinh \theta / 2] \sqrt{r^{2}+1}, \\
& X_{2}^{\mathrm{AdS}_{3}}=[\sin t / 2 \sinh u / 2 \cosh \theta / 2-\cos t / 2 \cosh u / 2 \sinh \theta / 2] \sqrt{r^{2}+1}, \\
& X_{3}^{\mathrm{AdS}_{3}}=[\sin t / 2 \cosh u / 2 \sinh \theta / 2+\cos t / 2 \sinh u / 2 \cosh \theta / 2] \sqrt{r^{2}+1}, \\
& X_{4}^{\mathrm{AdS}_{3}}=r .
\end{aligned}
$$

The metric is now foliated by $\mathrm{AdS}_{3}$ space-times,

$$
d s_{\mathrm{AdS}_{3}}^{2}=\frac{d r^{2}}{r^{2}+1}+\frac{\left(r^{2}+1\right)}{4}\left(-\cosh (\theta)^{2} d t^{2}+d \theta^{2}+(d u+\sinh \theta d t)^{2}\right)
$$

where $r \in[-\infty, \infty], \theta \in[-\infty, \infty], t \in[-\infty, \infty]$ and global AdS has $u \in[-\infty, \infty]$. The space-time is non-simply connected when $u$ is periodic, $u \in[0, a]$.

These two foliations are obviously related by a change of coordinates:

$$
\begin{aligned}
u & =\frac{1}{2} \ln \left(\frac{1+2 \rho^{2}-\rho^{2} y^{2}-2 \rho \cos (\tau-\phi) \sqrt{\left(1+\rho^{2}\right)\left(1-y^{2}\right)}}{1+2 \rho^{2}-\rho^{2} y^{2}+2 \rho \cos (\tau-\phi) \sqrt{\left(1+\rho^{2}\right)\left(1-y^{2}\right)}}\right), \\
\sinh \theta & =\frac{2 \rho \sin (\phi-\tau) \sqrt{\left(1+\rho^{2}\right)\left(1-y^{2}\right)}}{1+\rho^{2} y^{2}}, \\
\sin t & =\frac{\sin (2 \tau)\left(1+\rho^{2}\right)-\sin (2 \phi) \rho^{2}\left(1-y^{2}\right)}{\sqrt{\left(1+2 \rho^{2}-\rho^{2} y^{2}\right)^{2}-4 \rho^{2}\left(1-y^{2}\right)\left(1+\rho^{2}\right) \cos (\tau-\phi)^{2}}}, \\
r & =\rho y .
\end{aligned}
$$

It follows that positive $r$ corresponds to the northern hemisphere of the $\mathbb{S}^{2}$ and negative $r$ to the southern hemisphere in the sphere foliation. Therefore the boundary at $r>0$ $(y>0)$ should be in some form connected with the boundary at $r<0(y<0)$ through the $\mathbb{S}^{1}$ equator $(y=0)$ of the two hemispheres of the $\mathbb{S}^{2}$. Let us now study the $\rho=\infty$ asymptotic region in the $\mathrm{AdS}_{3}$ foliation. To this end, we shall consider the boundary change of coordinates

$$
\begin{aligned}
u & =\frac{1}{2} \ln \left(\frac{2-y^{2}+2 \cos (\tau-\phi) \sqrt{1-y^{2}}}{2-y^{2}-2 \cos (\tau-\phi) \sqrt{1-y^{2}}}\right), \\
\sinh \theta & =\frac{2 \sin (\phi-\tau) \sqrt{1-y^{2}}}{y^{2}}, \\
\sin t & =\frac{\sin (2 \tau)-\sin (2 \phi)\left(1-y^{2}\right)}{\sqrt{\left(2-y^{2}\right)^{2}-4\left(1-y^{2}\right) \cos (\tau-\phi)^{2}}} .
\end{aligned}
$$

We pick the natural representative of the conformal boundary of $\mathrm{AdS}_{4}$ in the foliation by $\mathrm{AdS}_{3}$ space-times (5.7) as given by the metric

$$
d s_{3 D}^{2}=\frac{1}{4}\left[-\cosh ^{2} \theta d t^{2}+d \theta^{2}\right]+\frac{1}{4}(d u+\sinh \theta d t)^{2},
$$


which is globally $\mathrm{AdS}_{3}$ space-time if all the coordinates cover the real line. If $u \sim u+a$ and the other coordinates cover the whole real line this three dimensional background is the Coussaert-Henneaux space-time. If we plug the change of coordinates (5.12)-(5.14) in $(5.15)$ we get

$$
d s_{3 D}^{2}=y^{-2}\left(-d \tau^{2}+\frac{d y^{2}}{1-y^{2}}+\left(1-y^{2}\right) d \phi^{2}\right) .
$$

Indeed, it can be seen from the boundary point of view that the region of $\theta= \pm \infty$ of (5.15) corresponds to $y=0$. This is a surface with the topology of $S^{1} \times \mathbb{R}$, which is the usual conformal boundary of $\mathrm{AdS}_{3}$ and also the border between the two hemispheres of the usual conformal boundary of $\mathrm{AdS}_{4}, \mathbb{S}^{2} \times \mathbb{R}$.

Here we want to remark a very interesting subtlety. As is well known, AdS does not have a boundary but a conformal boundary. The representative of the conformal boundary given by (5.15) is in a different equivalence class than $\mathbb{S}^{2} \times \mathbb{R}$. This can be seen directly from (5.16) as the conformal factor that relate these two metrics is singular at $y=0$. Therefore, we conclude that the slicing of $\mathrm{AdS}_{4}$ by $\mathrm{AdS}_{3}$ has two connected $\mathrm{AdS}_{3}$ boundaries if we pick the boundary representative in the equivalence class of (5.15) and one boundary if we pick the representative

$$
d \bar{s}_{3 D}^{2}=\omega(u, \theta)^{2} d s_{3 D}^{2}=\frac{\omega(u, \theta)^{2}}{4}\left[-\cosh ^{2} \theta d t^{2}+d \theta^{2}\right]+\frac{\omega(u, \theta)^{2}}{4}(d u+\sinh \theta d t)^{2},
$$

where

$$
\omega(u, \theta)^{2}=\frac{1}{1-\left(\cosh \frac{\theta}{2}-\cosh \frac{u}{2}\right)^{2}} .
$$

Let us now pass to study a representative of the conformal boundary of the wormhole space-time (3.1), given by the metric

$$
d s_{\partial M}^{2}=\frac{1}{4}\left[-\cosh ^{2} \theta d t^{2}+d \theta^{2}\right]+\frac{1}{\sigma}(d u+\sinh \theta d t)^{2},
$$

which is a quotient of space like warped $\mathrm{AdS}_{3}$ when $\sigma \neq 4$. The quotient is product of the identification $u \sim u+a$. If we plug the change of coordinates (5.12)-(5.14) in (5.19) we get

$$
\begin{aligned}
d s_{\partial M}^{2}= & y^{-2}\left(-d \tau^{2}+\frac{d y^{2}}{1-y^{2}}+\left(1-y^{2}\right) d \phi^{2}\right) \\
& +y^{-2}\left(\frac{4}{\sigma}-1\right)\left(1-y^{2}\right)\left[\cos (\tau-\phi) \frac{d y}{1-y^{2}}-y^{-1} \sin (\tau-\phi)(d \tau+d \phi)\right]^{2} .
\end{aligned}
$$

For $\sigma=4$, this is exactly the same boundary than the locally $\mathrm{AdS}_{4}$ we just discussed, so we shall consider from now on only the $\sigma \neq 4$ case. The first important aspect of this metric is that $y^{2} d s_{\partial M}^{2}$ is not locally $\mathbb{S}^{2} \times \mathbb{R}$. Moreover, an straightforward calculation of the Riemann tensor of (5.19) yields

$$
R_{t \theta}^{t \theta}=4\left(1-3 \sigma^{-1}\right), \quad R_{t u}^{t u}=R_{\theta u}^{\theta u}=4 \sigma^{-1}, \quad R_{t \theta}^{\theta u}=4 \sinh \theta\left(1-4 \sigma^{-1}\right) .
$$

Hence, from (5.21) we observe that warped $\mathrm{AdS}_{3}$ develops a parallelly propagated curvature singularity (PPS) at $\theta= \pm \infty$, we discuss this in more detail in the section on geodesics 
below. One could insist in try to construct a space-time with a single boundary by picking a metric representative in the class of $y^{2} d s_{\partial M}^{2}$. However this metric has a curvature singularity. An straightforward computation of the Ricci scalar yields

$$
R\left(y^{2} d s_{\partial M}^{2}\right)_{\theta= \pm \infty}=2\left(1-4 \sigma^{-1}\right) e^{|\theta|}+O\left(e^{|\theta| / 2}\right)
$$

Therefore, the two warped AdS boundaries cannot be joined in a single compact boundary by a conformal transformation.

\subsection{Geodesics and a parallel propagating singularity}

Our wormhole avoids the use of matter violating the null energy condition because the throat has a minimal $\mathbb{S}^{1}$ instead of a minimal $\mathbb{S}^{2}$ as required in [5]. We would like to discuss here the different families of null geodesics and use them to clarify the structure of the conformal boundary just described. A representative of the conformal boundary of the wormhole space-time (3.1) is given by the metric

$$
d s_{\partial M}^{2}=\frac{\ell^{2}}{4}\left[-\cosh ^{2} \theta d t^{2}+d \theta^{2}\right]+\frac{\ell^{2}}{\sigma}(d u+\sinh \theta d t)^{2},
$$

and we set again $\ell=1$. Let us pass to study its geodesics. This space-time has four Killing vectors (3.11), which yield four conserved charges along geodesic motion

$$
Q_{[i]}=4 \dot{x}^{\mu} \xi_{\mu[i]}
$$

where $\dot{x}^{\mu}=(\dot{t}, \dot{\theta}, \dot{u})$. These charges allow to find the velocities

$$
\begin{aligned}
\dot{t} & =-Q_{1}+L \tanh \theta \sin \left(t-t_{0}\right) \\
\dot{\theta} & =L \cos \left(t-t_{0}\right) \\
\dot{u} & =-L \frac{\sin t}{\cosh \theta}+\left(\frac{\lambda^{2}-1}{\lambda^{2}}\right)\left(Q_{1} \sinh \theta+L \cosh \theta \sin \left(t-t_{0}\right)\right)
\end{aligned}
$$

where $\lambda^{2}=\frac{4}{\sigma}, Q_{2}=L \sin t_{0}$ and $Q_{3}=L \cos t_{0}$ and consistency of (3.1) requires $Q_{1}=Q_{4}$. The condition that the geodesic is null yields

$$
\begin{aligned}
0= & g_{\mu \nu} \dot{x}^{\mu} \dot{x}^{\nu} \\
= & \frac{\left(1-\lambda^{2}\right)}{4 \lambda^{2}}\left[\left(L^{2} \sin ^{2}\left(t-t_{0}\right)+Q_{1}^{2}\right) \cosh ^{2} \theta+L Q_{1} \sinh 2 \theta \sin \left(t-t_{0}\right)\right] \\
& +\frac{L^{2} \lambda^{2}-Q_{1}^{2}}{4 \lambda^{2}}
\end{aligned}
$$

plugging (5.26) in (5.28) we obtain an equation in terms of $\dot{\theta}$ and functions of $\theta$ only. It has two solutions for $\dot{\theta}^{2}$

$$
\dot{\theta}_{ \pm}^{2}=\frac{L^{2}-Q_{1}^{2}}{L^{2}}+\frac{\lambda^{2} L^{2}+Q_{1}^{2}-2 \lambda^{2} Q_{1}^{2}}{L^{2}\left(1-\lambda^{2}\right) \cosh ^{2} \theta} \pm \frac{\sin \theta}{\cosh ^{2} \theta} \frac{\lambda Q_{1}}{L^{2}} \sqrt{\frac{Q_{1}^{2}-L^{2}}{1-\lambda^{2}}}
$$


Supersymmetric wormholes have $\lambda \leq 1$. The case with $\lambda=1$ is special as it corresponds to locally $\mathrm{AdS}_{3}$ space-time and we will discuss it below. When $\lambda<1$ the integrals of motion must satisfy $Q_{1}^{2}-L^{2} \geq 0$ for the square root to be a real number in (5.29). This in turn implies that the motion is confined. Indeed, when $Q_{1}^{2}-L^{2} \geq 0$ we see that $\dot{\theta}_{ \pm}^{2}<0$ for large enough $|\theta|$, which implies that existence of a turning point at some point when $\dot{\theta}_{ \pm}^{2}=0$. Hence, generic null geodesics are confined to live at bounded $\theta$.

There is an exception. Namely, whenever $L^{2}-Q_{1}^{2}=0$. When we replace the condition $Q_{1}= \pm L$ in (5.28) yields

$$
\theta= \pm \frac{1}{2} \ln \left(\frac{1-\sin \left(t-t_{0}\right)}{1+\sin \left(t-t_{0}\right)}\right)
$$

which allows to integrate the remaining geodesic equation

$$
u=u_{0} \pm \ln \left(\cos \left(t-t_{0}\right)\right) \text {. }
$$

We conclude that this geodesic reaches $\theta= \pm \infty$ at $t-t_{0}=\frac{\pi}{2}$. Note that the condition $\lambda=1$ also enforces $L^{2}-Q_{1}^{2}=0$ in (5.28). Now, we shall use this geodesic to construct a parallelly propagated orthonormal frame (PPO). If the geodesic that reaches null infinity has tangent vector $X^{\mu}$, a set of vierbeine where one is aligned along $X^{\mu}$ will define such a basis. So we pick $\hat{e}_{\mu}^{0}=X_{\mu}$. In this way we construct the Riemann tensor in this basis

$$
R_{a b c d}^{P P}=R^{\mu \nu \delta \alpha} \hat{e}_{a \mu} \hat{e}_{b \nu} \hat{e}_{c \delta} \hat{e}_{d \alpha}
$$

If the Ricci tensor is singular in the PPO then it implies that the Riemann is singular which is the definition of a PPS. We will only need to compute one component of the Ricci tensor in the PPO to check that this is the case:

$$
R_{\mu \nu} X^{\mu} X^{\nu}=\eta^{a c} R_{a 0 c 0}^{P P}=2 L^{2} \sinh ^{2}(\theta)\left(\lambda^{2}-1\right) .
$$

Hence, we see that unless $\lambda=1$, tidal forces diverge when $\theta \longrightarrow \pm \infty$. Note however that all curvature components are constant in a static frame

$$
E^{0}=\cosh \theta d t, \quad E^{1}=d \theta, \quad E^{2}=d u+\sinh \theta d t
$$

since the Riemann tensor for warped AdS is

$$
R_{0202}=3 \lambda^{2}-4, \quad R_{0303}=-1, \quad R_{2323}=1,
$$

where we recall that we are using a convention where the Riemann tensor of AdS is constant and positive.

Hence, we conclude that when $\sigma=4$, the boundaries can be connected by null geodesics. These geodesics can go from one boundary to the other without problem. Moreover, the time it takes a geodesic to connect the boundary through the bulk (which has a minimum at $t=2 \pi$ ) is at least the double it takes through the boundary. Furthermore, it follows from (5.31) that this null geodesic winds around the $u$ coordinate infinite many times. The curves that winds around $u$ are non contractible. Hence, the geodesic along the 
boundary is not-homotopic to the geodesic along the bulk. As expected in a non-simply connected wormhole space-time.

When $\sigma \neq 4$, it is impossible to connect the different boundaries with geodesics due to the presence of a PPS. This singularity is a property of the warped AdS space-time that seems to have passed unnoticed so far in the literature, see for instance [22].

\section{Topological censorship}

There is a set of results that seem to indicate that wormholes can not exist. In particular in [12] it is stated the following theorem: let $\mathcal{M}$ be a globally hyperbolic space-time-withboundary with timelike boundary $\mathcal{I}$ that satisfies averaged null energy condition. Let $\mathcal{I}_{0}$ be a connected component of $\mathcal{I}$ of $\mathcal{M}$. Furthermore assume that either (i) $\mathcal{I}_{0}$ admits a compact spacelike cut or (ii) $\mathcal{M}$ satisfies the generic condition. Then $\mathcal{I}_{0}$ cannot communicate with any other component of $\mathcal{I}$.

This is particularly relevant to us, as the wormhole seems to have a boundary with two disconnected components when $\sigma \neq 4$. The boundary, given by warped $\mathrm{AdS}_{3}$, has non-compact spacelike surfaces. So we need to verify that the generic condition is not satisfied. The generic condition is the statement that for every timelike or null geodesic $Z_{\mu}$ there is a point where $Z_{[\sigma} R_{\mu] \nu \lambda[\alpha} Z_{\beta]} Z^{\nu} Z^{\lambda} \neq 0$.

It is possible to verify that, when the gauge field vanishes, our wormhole spacetime

$$
d s^{2}=\frac{4 \ell^{4} d r^{2}}{\sigma^{2} f(r)}+h(r)\left[-\cosh ^{2} \theta d t^{2}+d \theta^{2}\right]+f(r)(d u+\sinh \theta d t)^{2},
$$

with metric functions

$$
f(r)=\frac{2}{q^{2} \sigma^{2}} \frac{r^{4}+(6-\sigma) r^{2}+m r+\sigma-3}{r^{2}+1}, \quad h(r)=\frac{1}{2 q^{2} \sigma}\left(r^{2}+1\right),
$$

satisfies

$$
Z_{[\sigma} R_{\mu] \nu \lambda[\alpha} Z_{\beta]} Z^{\nu} Z^{\lambda}=0
$$

for the following null geodesic

$$
Z=\frac{1}{h(r) \cosh ^{2} \theta} \partial_{t}-\frac{1}{h(r) \cosh \theta} \partial_{\theta}-\frac{\sinh \theta}{h(r) \cosh ^{2} \theta} \partial_{u} .
$$

Note that the causal structure of the space-time is the same whether there is an electromagnetic field or not. Hence, our wormhole avoids topological censorship by not being within the hypothesis of the theorem.

\section{Conclusion}

In this paper we considered supersymmetric transversable wormholes that are everywhere regular with and without electromagnetic fields. In this respect these wormholes are crucially different from black holes, which have a curvature singularity in the interior of the event horizon. The supersymmetric wormholes preserve half of the supersymmetries. An 
interesting fact is that this situation also exists in an AdS space upon the introduction of a non-contractible cycle. In that case there exist potentially eight Killing spinors, but only half of them are globally defined, as was shown in equation (4.8). The supersymmetry algebra then implies that the Killing vectors of this space-time exhibit the same feature, namely that some of them will not be globally defined. Note, however, that the latter scenario does not involve electromagnetic fields.

It is worth mentioning that wormhole geometries in asymptotically AdS space-times have received attention in connection with holography (see e.g. [23]) The presence of multiple boundaries would then create the possibility of couplings between different CFTs. It had already been noted earlier that the interaction between two CFTs opens a throat in the bulk that causally connects the two boundaries [24]. However, most of these settings require non-local interactions between the boundaries for the wormhole throat to open, a feature that is not present in the construction of this paper.

The supersymmetric, charged, transversable wormholes provide a concrete physical realization of the Weyl's idea referred to in the introduction. Non-trivial electromagnetic field lines can be supported by a geometry that is consistent with the Einstein-Maxwell system.

\section{Acknowledgments}

We thank Nava Gaddam, Adolfo Guarino, Mario Trigiante and Antoine Van Proeyen for valuable discussions. We also would like to thank an anonymous referee who motivate us to include the analysis of sections 5.1 and 5.2. We wish to thank the project MEC80170073 of CONICYT, Chile, which made this work possible. We would like to thank the support of Proyecto de cooperación internacional 2019/13231-7 FAPESP/ANID. The research of AA is supported in part by the Fondecyt Grants 1170279 and 1161418 and the Alexander von Humboldt Foundation. The research of JO is supported in part by the Fondecyt Grant 1181047. AA wishes to thank to the warm hospitality at the Albert Einstein Institut, Golm, Germany and at the Institute for Theoretical Physics, Utrecht University, Netherlands, where part of this work was carried out.

Open Access. This article is distributed under the terms of the Creative Commons Attribution License (CC-BY 4.0), which permits any use, distribution and reproduction in any medium, provided the original author(s) and source are credited.

\section{References}

[1] R.W. Fuller and J.A. Wheeler, Causality and Multiply Connected Space-Time, Phys. Rev. 128 (1962) 919 [INSPIRE].

[2] A. Einstein and N. Rosen, The Particle Problem in the General Theory of Relativity, Phys. Rev. 48 (1935) 73 [INSPIRE].

[3] M.S. Morris, K.S. Thorne and U. Yurtsever, Wormholes, Time Machines, and the Weak Energy Condition, Phys. Rev. Lett. 61 (1988) 1446 [INSPIRE].

[4] M.S. Morris and K.S. Thorne, Wormholes in space-time and their use for interstellar travel: A tool for teaching general relativity, Am. J. Phys. 56 (1988) 395 [INSPIRE]. 
[5] D. Hochberg and M. Visser, The Null energy condition in dynamic wormholes, Phys. Rev. Lett. 81 (1998) 746 [gr-qc/9802048] [INSPIRE].

[6] E. Witten and S.-T. Yau, Connectedness of the boundary in the AdS/CFT correspondence, Adv. Theor. Math. Phys. 3 (1999) 1635 [hep-th/9910245] [INSPIRE].

[7] J.M. Maldacena and L. Maoz, Wormholes in AdS, JHEP 02 (2004) 053 [hep-th/0401024] [INSPIRE].

[8] P. Breitenlohner and D.Z. Freedman, Positive Energy in anti-de Sitter Backgrounds and Gauged Extended Supergravity, Phys. Lett. B 115 (1982) 197 [InSPIRE].

[9] P. Breitenlohner and D.Z. Freedman, Stability in Gauged Extended Supergravity, Annals Phys. 144 (1982) 249 [INSPIRE].

[10] A. Anabalón and J. Oliva, Four-dimensional Traversable Wormholes and Bouncing Cosmologies in Vacuum, JHEP 04 (2019) 106 [arXiv:1811.03497] [INSPIRE].

[11] J. Maldacena, A. Milekhin and F. Popov, Traversable wormholes in four dimensions, arXiv: 1807.04726 [INSPIRE].

[12] G.J. Galloway, K. Schleich, D. Witt and E. Woolgar, The AdS/CFT correspondence conjecture and topological censorship, Phys. Lett. B 505 (2001) 255 [hep-th/9912119] [INSPIRE].

[13] D.Z. Freedman and A.K. Das, Gauge Internal Symmetry in Extended Supergravity, Nucl. Phys. B 120 (1977) 221 [INSPIRE].

[14] E.S. Fradkin and M.A. Vasiliev, Model of supergravity with minimal electromagnetic interaction, Lebedev Institute preprint (1976) [INSPIRE].

[15] B. de Wit, J.W. van Holten and A. Van Proeyen, Structure of $N=2$ Supergravity, Nucl. Phys. B 184 (1981) 77 [Erratum ibid. 222 (1983) 516] [INSPIRE].

[16] B. de Wit and A. Van Proeyen, Potentials and Symmetries of General Gauged $N=2$ Supergravity: Yang-Mills Models, Nucl. Phys. B 245 (1984) 89 [INSPIRE].

[17] B. de Wit, P.G. Lauwers and A. Van Proeyen, Lagrangians of $N=2$ Supergravity-Matter Systems, Nucl. Phys. B 255 (1985) 569 [inSPIRE].

[18] L.J. Romans, Supersymmetric, cold and lukewarm black holes in cosmological Einstein-Maxwell theory, Nucl. Phys. B 383 (1992) 395 [hep-th/9203018] [InSPIRE].

[19] O. Coussaert and M. Henneaux, Selfdual solutions of $(2+1)$ Einstein gravity with a negative cosmological constant, in The Black Hole 25 Years After, pp. 25-39 (1994) [hep-th/9407181] [INSPIRE].

[20] H. Lü and J. Mei, Ricci-Flat and Charged Wormholes in Five Dimensions, Phys. Lett. B 666 (2008) 511 [arXiv:0806.3111] [INSPIRE].

[21] P.K. Townsend, Surprises with angular momentum, Annales Henri Poincaré 4 (2003) S183 [hep-th/0211008] [INSPIRE].

[22] I. Bengtsson and P. Sandin, Anti de Sitter space, squashed and stretched, Class. Quant. Grav. 23 (2006) 971 [gr-qc/0509076] [INSPIRE].

[23] J. Maldacena and X.-L. Qi, Eternal traversable wormhole, arXiv:1804.00491 [INSPIRE].

[24] P. Gao, D.L. Jafferis and A.C. Wall, Traversable Wormholes via a Double Trace Deformation, JHEP 12 (2017) 151 [arXiv: 1608.05687] [INSPIRE]. 